\title{
Evaluación de t-MOOC universitario sobre competencias digitales docentes mediante juicio de expertos según el Marco DigCompEdu
}

\section{Evaluation of university t-MOOC on teaching digital competences through expert judgment according to the DigCompEdu Framework}

\author{
Julio Cabero-Almenara \\ Universidad de Sevilla, Sevilla, España \\ cabero@us.es \\ Julio Barroso... Osuna \\ Universidad de Sevilla, Sevilla, España \\ jbarroso@us.es \\ Antonio Palacios--Rodríguez \\ Universidad de Sevilla, Sevilla, España \\ aprodriguez@us.es \\ Carmen Llorente...Cejudo \\ Universidad de Sevilla, Sevilla, España \\ karen@us.es
}

\begin{abstract}
RESUMEN
Los MOOC se configuran como una de las tecnologías que, en los últimos tiempos, vienen ganando terreno en el campo educativo como nuevos enfoques en la educación a distancia, incrementándose su presencia en las instituciones educativas y el nivel de investigaciones y publicaciones que giran alrededor de estos desarrollos tecnológicos. En concreto, esta investigación se centra en el t-MOOC basado en la tarea. Se presenta la evaluación de un t-MOOC diseñado y producido para el desarrollo de competencias digitales del profesorado universitario a través del juicio de expertos según el Marco DigCompEdu. En su evaluación participan 172 expertos que obtienen un índice de competencia experta igual o superior a 0,9. Las puntuaciones alcanzadas permiten señalar que los expertos valoraron el t-MOOC de manera muy positiva, tanto de forma conjunta como individual. Igualmente, el estudio pone de manifiesto la significación del Coeficiente de Competencia Experta (CCE) a la hora de discriminar de forma más adecuada y justificada la selección de los expertos. Por este motivo, se reflexiona sobre las posibilidades didácticas que este tipo de tecnología añade a los nuevos escenarios educativos.
\end{abstract}

Palabras clave: Competencia digital; DigCompEdu; T-MOOC; diseño de medios y materiales; índice de competencia experta.

RED. Revista de Educación a Distancia. Núm. 66, Vol. 21. Artíc. 2, 30-Sep-2021

DOI: http://dx.doi.org/10.6018/red.476891 


\begin{abstract}
MOOCs are configured as one of the technologies that in recent times have been gaining ground in the educational field as new approaches in distance education, increasing their presence in educational institutions and the level of research and publications that revolve around these developments technological. Specifically, this research focused on the task-based t-MOOC. The evaluation of a t-MOOC carried out for the development of digital competences (DigCompEdu model) of university teachers is presented through the judgment of experts. 172 experts participate in its evaluation, obtaining an expert competence index equal to or greater than 0.9 . The scores achieved allow us to indicate that the experts valued the t-MOOC very positively, both jointly and individually. Likewise, the study shows the significance of the Expert Competence Coefficient (ECC) when it comes to discriminating in a more adequate and justified way the selection of experts. For this reason, we reflect on the didactic possibilities of this type of technology in the new educational settings.

Key words: Digital competence; DigCompEdu; T-MOOC; media and material design; expert proficiency index.
\end{abstract}

\title{
LOS MOOC COMO TECNOLOGÍA FORMATIVA
}

Una de las tecnologías que ha ido ganando terreno progresivamente en los últimos tiempos han sido los MOOC como un nuevo enfoque de la educación a distancia. Los MOOC surgen a partir del movimiento de recursos educativos abiertos (Pilli y Admiraal, 2016). Están incrementando su presencia en las instituciones educativas y su volumen de investigaciones y publicaciones sobre ellos es cada vez mayor (Bozkurt et al., 2016; RuizPalmero et al., 2021). Algunas investigaciones han puesto de manifiesto que tienen un fuerte potencial como herramientas educativas en general, como recursos para el aprendizaje y son de gran utilidad para la formación permanente de las personas (García Aretio, 2017; Benet et al., 2018; Palacios et al., 2020). Además, específicamente, son de gran utilidad para el desarrollo profesional de los docentes (Castaño-Muñoz et al., 2018). Estas líneas de investigación, tal y como sugieren Zawacki-Richer et al. (2018), se han centrado fundamentalmente en cuatro líneas de investigación: (a) el potencial y los desafíos de los MOOC para las universidades; (b) plataformas MOOC; (c) estudiantes y contenido en MOOC; y (d) la calidad de los MOOC y los problemas de diseño instruccional.

También es necesario apuntar que a los MOOC se les ha concedido una serie de ventajas e inconvenientes (Tabla 1).

Evaluación de t-MOOC sobre competencias digitales docentes universitario mediante el juicio de expertos. Selección mediante el coeficiente de competencia experta. Cabero-Almenara, BarrosoOsuna, Palacios-Rodríguez, y Llorente-Cejudo. 
RED. Revista de Educación a Distancia. Núm. 67, Vol. 21. Artíc. 2, 30-Sep-2021

DOI: http://dx.doi.org/10.6018/red.476891

Tabla 1. Ventajas e inconvenientes de los MOOC, según Fernández-Ferrer (2019).

\begin{tabular}{|c|c|c|}
\hline & Ventajas & Inconvenientes \\
\hline \multirow[t]{14}{*}{ Estudiantes } & Acceso gratuito & Poca atención a las individualidades \\
\hline & Acreditación & Seguimiento y feedback del profesorado \\
\hline & Educación a lo largo de la vida & inadecuado \\
\hline & Apertura & Requisitos previos al curso \\
\hline & Especialización & Costes adicionales \\
\hline & Recursos y contenidos de alta calidad & Baja participación \\
\hline & Posibilidades hacia la educación superior & Bajo reconocimiento \\
\hline & Flexibilidad & Funcionamiento \\
\hline & Universidades y profesores de prestigio & Abandono \\
\hline & Aprendizaje colaborativo & \\
\hline & $\begin{array}{l}\text { Aprendizaje autónomo y adquisición de } \\
\text { competencias }\end{array}$ & \\
\hline & $\begin{array}{l}\text { Adecuación a las necesidades formativas } \\
\text { del momento }\end{array}$ & \\
\hline & Inserción laboral y empleo & \\
\hline & $\begin{array}{l}\text { Consecuencias prácticas para la } \\
\text { evaluación }\end{array}$ & \\
\hline \multirow[t]{4}{*}{ Profesores } & Visibilidad y reputación docente & Dificultades en la evaluación \\
\hline & Investigación e innovación docente & Elevada carga de trabajo \\
\hline & & $\begin{array}{l}\text { Falta de conexión con los nuevos roles } \\
\text { docentes }\end{array}$ \\
\hline & & Necesidades tecnológicas previas \\
\hline \multirow{6}{*}{$\begin{array}{l}\text { Instituciones } \\
\text { Educación } \\
\text { Superior }\end{array}$} & Inversión o reducción de costes & Costes \\
\hline & Aumento de los estudiantes & Prácticas fraudulentas \\
\hline & Internacionalización & Problemas técnicos \\
\hline & Marketing & Inexistencia de evidencias y resultados \\
\hline & $\begin{array}{l}\text { Experimentación e innovación Cambios y } \\
\text { mejoras de la oferta académica }\end{array}$ & $\begin{array}{l}\text { Necesidad de actualización de los } \\
\text { recursos }\end{array}$ \\
\hline & Adaptación a las demandas del momento & \\
\hline
\end{tabular}

Fuente: Elaboración propia.

Inicialmente, estas herramientas educativas vinieron marcadas por una serie de características básicas: se desarrollan de manera on-line, son abiertos, son gratuitos para su realización no así para su certificación y acreditación, movilizan un amplio número de estudiantes, no requieren requisitos de ingreso y admisión y permiten una participación interactiva de un gran volumen de estudiantes (Castaño \& Cabero, 2013; Castaño-Muñoz et al., 2018).

Para Escudero-Nahón \& Núñez-Urbina (2020, p.190), en el trabajo que realizan sobre la conceptualización de los MOOC, se señala que "desde un punto de vista educativo, los MOOC son programas inscritos en el ámbito del e-learning. Esta precisión es relevante porque este último tiene como principios educativos el uso de Internet para acceder a

Evaluación de t-MOOC sobre competencias digitales docentes universitario mediante el juicio de expertos. Selección mediante el coeficiente de competencia experta. Cabero-Almenara, BarrosoOsuna, Palacios-Rodríguez, y Llorente-Cejudo. 
recursos digitalizados, el aprendizaje en red, y la comunicación horizontal entre pares y docentes". Los autores también llaman la atención que la característica de "masivo", determinante en los primeros momentos, debe ser contempladas con cierta precaución en la actualidad.

Una de las principales críticas que se le ha asignado a los MOOC ha sido la fuerte deserción y abandono de los cursos por parte de los estudiantes. Dicho abandono disminuye cuando los alumnos han tenido una experiencia previa con estos tipos de cursos, tienen un alto auto compromiso para su realización (Albelbisi, et al., 2018; Deng et al., 2020) y presentan una actitud positiva hacia ellos (Zhou, 2016). Respecto al compromiso, Deng et al. (2020) señalan que existen diferentes tipos: emocional, conductual, social y cognitivo. Por otra parte, diferentes trabajos ponen de manifiesto que la edad de los alumnos y su nivel educativo repercutió en obtener mejores niveles de logro y, a su vez, fueron también los que más participaron en el foro de discusión (Mercado-del-Collado et al., 2019; Rocha et al., 2020).

Se debe señalar que el desarrollo de los MOOC ha traído como consecuencia la aparición de más variantes de los modelos iniciales de xMOOC y cMOOC, que presentaban formas de diseño completamente diferentes. Los primeros, preocupados en que los estudiantes adquieran una serie de contenidos. Por otra parte, los segundos en los cuales el conocimiento no se centra en los contenidos aportados por los expertos, sino más bien en las conexiones que son capaces de establecer los estudiantes que participan en el entorno formativo.

En este contexto aparecen nuevas configuraciones como los hMOOC, que son modelos híbridos entre los xMOOC y los CMOOC (García-Peñalvo et al., 2018); los bMOOC, que persigue combinar las ventajas del aprendizaje en línea y la interacción cara a cara (Zhao \& Song, 2020); los SPOOC, que se caracterizan por las restricciones tanto de acceso, como de número de participantes, fomentando de esta manera un aprendizaje más personalizado y práctico (Aguayo y Bravo, 2017).

Pilli \& Admiraal (2016) realizaron una taxonomía de los diferentes MOOC en función de dos dimensiones: masividad y apertura, y en función de ellos construyen una matriz bidimensional con cuatro categorías: (i) pequeña escala y menos abierta, (ii) pequeña escala y más abierta, (iii) gran escala y menos abierta, (iv) gran escala y más abierta (Figura 1).

Evaluación de t-MOOC sobre competencias digitales docentes universitario mediante el juicio de expertos. Selección mediante el coeficiente de competencia experta. Cabero-Almenara, BarrosoOsuna, Palacios-Rodríguez, y Llorente-Cejudo. 


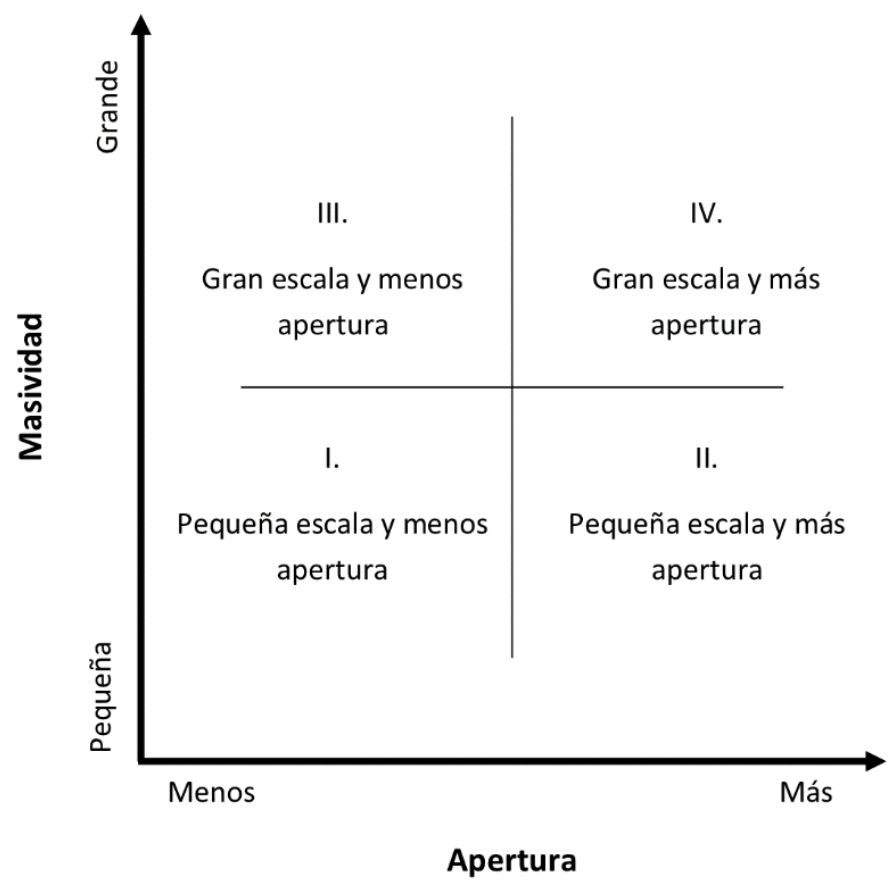

Figura 1. Propuesta de clasificación de los MOOC

Fuente: Pilli \& Admiraal (2016).

Dentro de los diferentes tipos de MOOC se encuentra el modelo seleccionado para este estudio, los denominados t-MOOC. Estos se basan en las tareas que deben realizar los estudiantes, de diferente tipología. Además, el alumnado debe realizar un mínimo de ellas para seguir avanzando en el curso y poder demostrar que domina las competencias que se desarrollan en dicho t-MOOC (Cabero et al., 2014; Osuna-Acedo et al., 2018). En paralelo, Pilli \& Admiraal (2016) afirman que este tipo de MOOC se apoyan en el instructivismo y el constructivismo.

Una de las ventajas que presenta este tipo de MOOC es que el estudiante debe tener una participación activa en el proceso educativo. Por otra parte, como han sugerido Albelbisi et al. (2018), una de las variables de éxito de los MOOC es la evaluación. Dicha evaluación se convierte en una variable crítica en este formato de MOOC para que el sujeto vaya avanzando en la acción formativa. Finalmente, como señalan diferentes autores, los MOOC son una excelente estrategia para la formación de los docentes en competencias digitales (Fernández et al., 2019; Gordillo et al., 2019).

Todos estos comentarios son los que han llevado a los investigadores de este trabajo a utilizarlos para la formación de los docentes universitarios bajo el Marco de Competencia Digital Docente DigCompEdu.

\section{El Marco de Competencia Digital Docente DigCompEdu}

La presencia de las tecnologías de la información y comunicación (TIC) en la formación universitaria hace que se esté acuñando nuevos términos como el de Competencia Digital Docente (CDD). Dicho término se refiere a la capacitación de los

Evaluación de t-MOOC sobre competencias digitales docentes universitario mediante el juicio de expertos. Selección mediante el coeficiente de competencia experta. Cabero-Almenara, BarrosoOsuna, Palacios-Rodríguez, y Llorente-Cejudo. 
docentes para el uso de las TIC en dimensiones más amplias que el simple manejo instrumental de ellas (Cabero-Almenara y Palacios-Rodríguez, 2020). La misma Comisión Europea apunta a que es una de las competencias significativas que deben dominar los estudiantes y los docentes para desenvolverse en los nuevos entornos tecnológicos formativos (Comisión Europea 2006).

Como señalan Flores \& Roig (2019), la CDD es multidimensional. Por ende, implica que la persona sea capaz de movilizar aquellas habilidades y destrezas que permiten buscar, seleccionar críticamente, obtener y procesar información relevante haciendo uso de las TIC. Todo ello para convertir la información identificada en conocimiento y saber comunicarnos con ellas de forma segura y responsable.

Este hecho ha llevado a que este tema de las CDD se esté convirtiendo en una línea consolidadade investigación en el contexto científico español (Rodríguez-García et al., 2018; Recio et al., 2020), que van desde su descripción, a la búsqueda de instrumentos de diagnóstico (Durán et al., 2016a; Tourón, et al., 2018; Padilla et al., 2019; Salazar \& Gibrán, 2019; Cabero-Almenara et al., 2020a), su certificación (Durán et al., 2016b; Amaya et al., 2018; Gudmundsdottir \& Hatlevic, 2018; Durán et al., 2019), su comparación entre diferentes marcos competenciales (Cabero-Almenara et al., 2020; Cabero-Almenara et al., 2021) y su grado adquirido por los docentes (CaberoAlmenara et al., 2020c; Ruiz et al., 2020; Cabero-Almenara, 2021; Infante-Moro et al., 2021).

En definitiva, como señala Durán (2019, p.27) es un "conjunto de conocimientos, capacidades y actitudes necesarios para que un docente haga un uso efectivo de las TIC desde sus distintas vertientes (tecnológica, informacional, multimedia, comunicativa, colaborativa y ética), asumiendo criterios pedagógico -didácticos para una integración efectiva de éstas en su experiencia docente".

Para la adquisición de estas CDD se han propuesto a nivel institucional diferentes marcos competenciales, como por ejemplo: "International Society for Technology in Education" (ISTE) para docentes, Unesco competencial TIC para docentes, el del Ministerio de Educación de Colombia y de Chile; que han sido analizados a través de distintos trabajos (Cabero \& Martínez, 2019; Lázaro et al., 2019; Padilla et al., 2019; Rodríguez-.García et al., 2019; Silva et al., 2019). En nuestro contexto va adquiriendo significación el propuesto por la Unión Europea y denominado "Marco Europeo de Competencia Digital del profesorado" o DigCompEdu (European Framework for the Digital Competence of Educators: DigCompEdu) (Comisión Europea, 2017; Cabero-Almenara \& PalaciosRodríguez, 2020). Este marco se articula alrededor de seis áreas competenciales: compromiso profesional, recursos digitales, pedagogía digital, evaluación y retroalimentación, empoderar a los estudiantes y facilitar la competencia digital a los estudiantes.

Al mismo tiempo, el DigCompEdu propone seis niveles competenciales progresivos: novato, explorador, integrador, experto, líder y pionero; que sugieren diferentes niveles de manejo e integración educativa de las tecnologías en la práctica educativa.

Evaluación de t-MOOC sobre competencias digitales docentes universitario mediante el juicio de expertos. Selección mediante el coeficiente de competencia experta. Cabero-Almenara, BarrosoOsuna, Palacios-Rodríguez, y Llorente-Cejudo. 


\section{METODOLOGÍA}

\section{Objetivo}

Esta investigación persigue la evaluación del t-MOOC realizado para la formación del profesorado universitario en la adquisición de las CDD según el marco competencia DigCompEdu. Esta investigación forma parte de otra más extensa denominada "Diseño, producción y evaluación de t-MOOC para la adquisición de competencias digitales del profesorado universitario" (tMOOCUNI). En ella, uno de los objetivos perseguidos es crear y evaluar un entorno formativo bajo la arquitectura t-MOOC para la capacitación del profesorado universitario en la adquisición de la CDD.

Al mismo tiempo, también pretende descubrir si las valoraciones realizadas por los expertos están determinadas por la titulación máxima que poseen, así como su lugar de trabajo.

\section{Características del t-MOOC producido}

Se presenta la evaluación de un t-MOOC producido para el desarrollo de la Competencia Digital Docente bajo el Marco DigCompEdu. La plataforma seleccionada para la construcción del t-MOOC ha sido Moodle. Tras la autentificación del docente y acceso al entorno, se le presentan dos animaciones iniciales; una, donde se explica al docente como debe desenvolverse en él; y otra, donde se presenta de forma general el modelo DigCompEdu y las diferentes competencias que lo conforman (Figura 2).

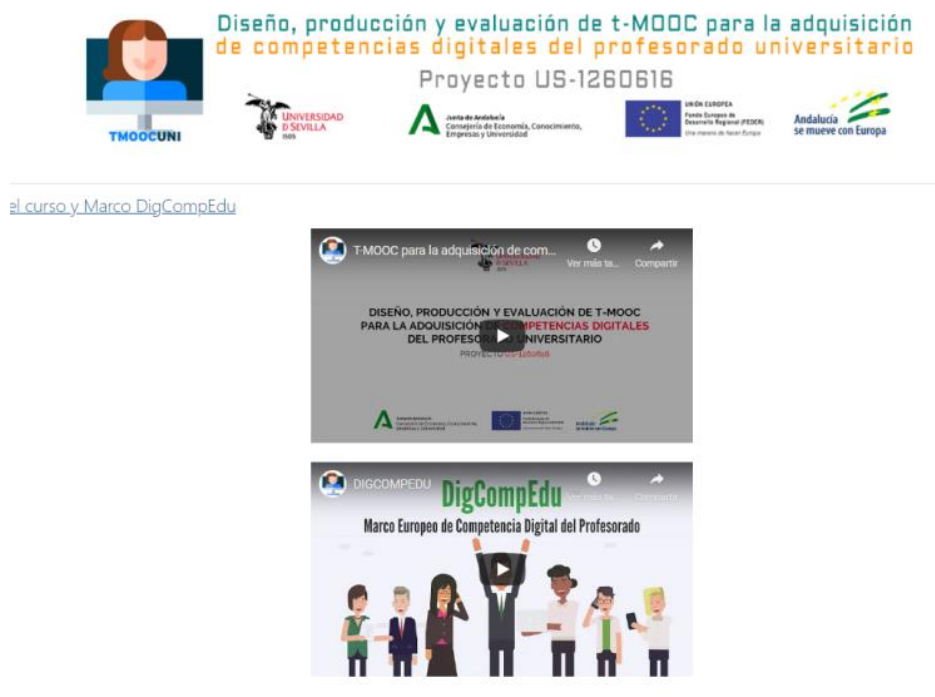

Figura 2. Presentación del t-MOOC.

Fuente: Elaboración propia.

La estructura del t-MOOC es la siguiente. Una vez observado los dos clips de vídeo, el docente se encuentra con la primera área competencial.

Evaluación de t-MOOC sobre competencias digitales docentes universitario mediante el juicio de expertos. Selección mediante el coeficiente de competencia experta. Cabero-Almenara, BarrosoOsuna, Palacios-Rodríguez, y Llorente-Cejudo. 


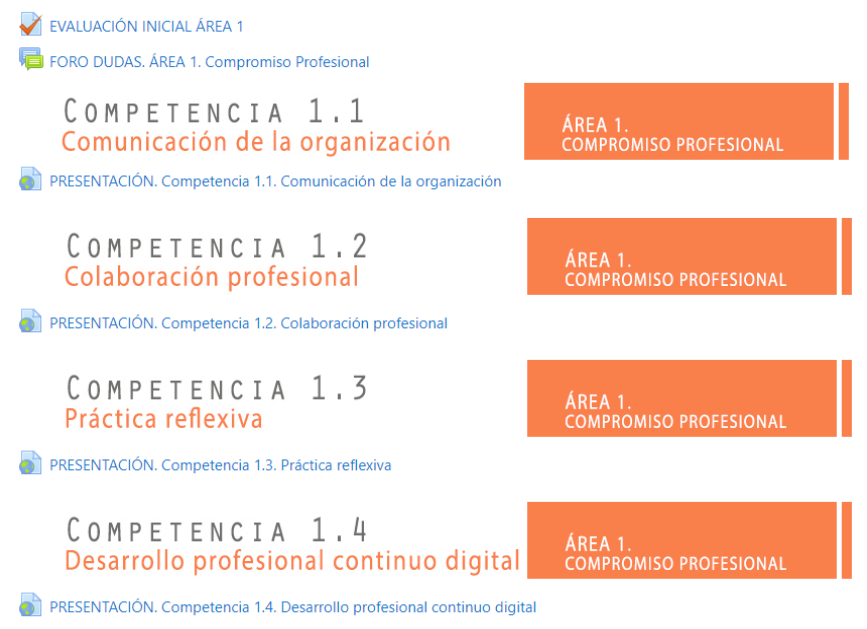

Figura 3. Presentación del área 1.

Fuente: Elaboración propia.

Se debe señalar que, para cada área competencial, el docente realiza una evaluación que indica el nivel que se posee: inicial, intermedio o avanzado (Figuras 4 y 5). De todas formas, siempre aparece un texto que recomienda revisar toda la información expuesta en los niveles inferiores al obtenido, aunque no tenga que realizar las tareas de estos niveles.

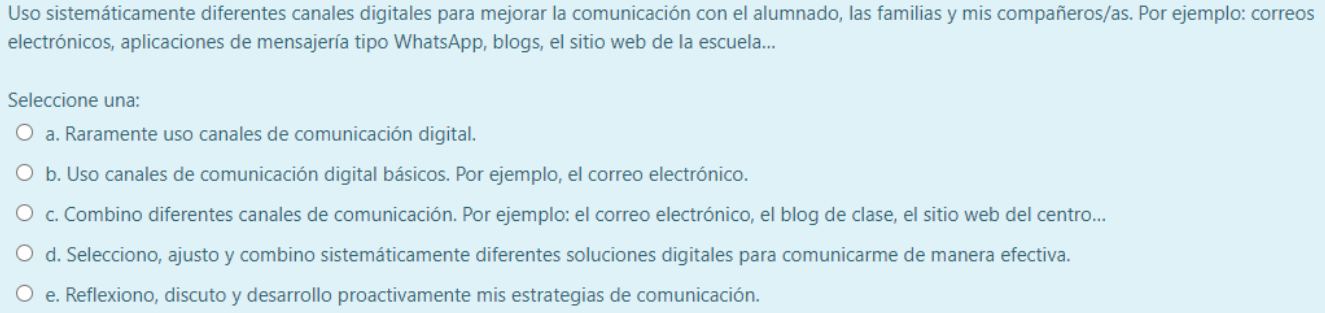

Figura 4. Ejemplo del cuestionario de evaluación por áreas.

Fuente: Elaboración propia.

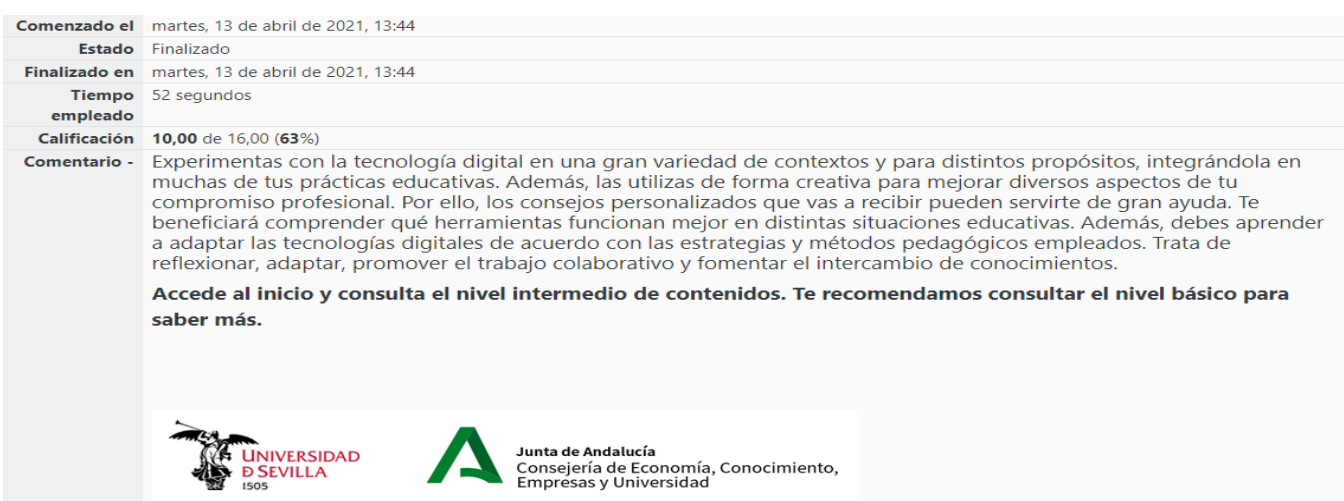

Figura 5. Ejemplo de feedback obtenido tras realizar el cuestionario de evaluación.

Fuente: Elaboración propia.

Evaluación de t-MOOC sobre competencias digitales docentes universitario mediante el juicio de expertos. Selección mediante el coeficiente de competencia experta. Cabero-Almenara, BarrosoOsuna, Palacios-Rodríguez, y Llorente-Cejudo. 
Al igual que el t-MOOC comienza con una animación, cada competencia comienza con un video didáctico que describe dicha competencia. Tras su visualización, el docente comienza con los contenidos del t-MOOC, y finaliza realizando las diferentes tareas. En concreto, se le ofrecen entre 4 a 6 actividades por competencia y nivel, de las cuales debe seleccionar 2 (Figura 6).

Tarea nivel inicial competencia 1.1

A continuación, le ofrecemos diferentes tareas que cubren este nivel (archivos PDF adjuntos).

Para superar la actividad, DEBE SELECCIONAR AL MENOS 2 TAREAS. Una vez las realice, debe subirlas al curso indicando el nombre de las tareas seleccionadas. Para ello, debe usar el boton "Agregar entrega" que se encuentra al final de esta página.

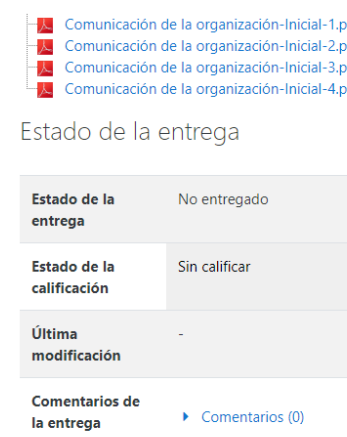

Agregar entrega

Figura 6. Pantalla de entrega de tareas.

Fuente: Elaboración propia.

La presentación de las tareas se efectúa mediante una guía donde se incorporan diferentes aspectos, tales como: su identificación, recomendaciones para su realización, lista de chequeo para que el docente compruebe la calidad de la entrega y una rúbrica de evaluación que es usada por los tutores del t-MOOC (Figura 7).

Evaluación de t-MOOC sobre competencias digitales docentes universitario mediante el juicio de expertos. Selección mediante el coeficiente de competencia experta. Cabero-Almenara, BarrosoOsuna, Palacios-Rodríguez, y Llorente-Cejudo. 


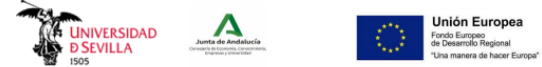

DISEÑO, PRODUCCIÓN Y EVALUACIÓN DE T-MOOC PARA LA ADQUISICIÓN DE COMPETENCIAS DIGITALES DEL PROFESOR UNIVERSITARIO

Nombre de la actividad: Localizar en esta sopa de letras creadas mediante Educoplay, cinco programas creadores de blog (Blogger, Jimdo, Blogia, Tumbir, Weebly) (Actividad 3) (Nivel Intermedio).

Competencia a la que pertenece: Comunicación de la organización.

Nivel: Intermedio.

Proyecto de Investigación: Proyecto US-1260616.

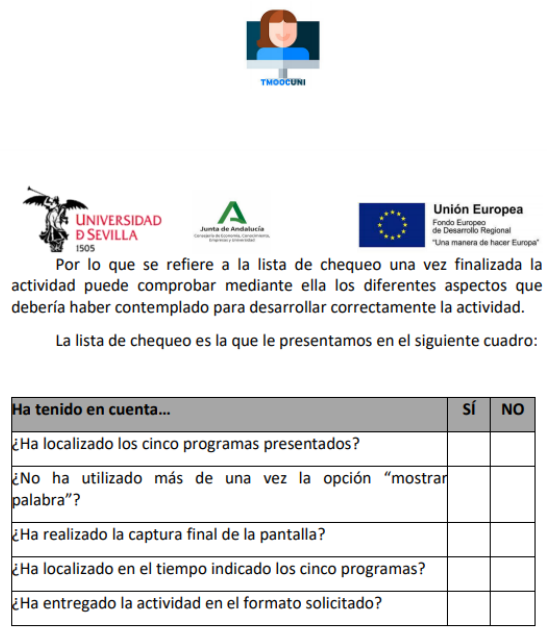

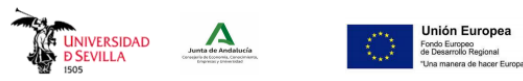

DESCRIPCIÓN DE LA ACTIVIDAD:

Localizar en esta sopa de letras creadas mediante Educoplay, cinco programas creadores de blog (Blogger, Jimdo, Blogia, Tumbir, Weebly). La url de la sopa de letras es: https://es.educaplay.com/recursoseducativos/8009518-programas generadores de blog.html. Dispone de 10 minutos para ello.

Para su realización le recomendamos que siga los siguientes pasos:

1) Lea bien los programas que debe buscar, ya que nada más entrar en el enlace el tiempo empezará a correr.

2) Entre en el enlace siguiente: https://es.educaplay.com/recursoseducativos/8009518-programas_generadores_de_blog.html.

3) Búsquelos en la sopa de letra. Arrastrando el ratón creará las palabras.

4) Si necesita alguna ayuda puede darle al botón: "Mostrar palabra". Pero ello debe hacerlo como máximo una vez.

5) Finalmente realice una captura de pantalla del resultado final alcanzado.

6) Envíe la actividad.

\section{FORMATO DE ENTREGA:}

Documento Word, writer, docs o pdf, donde incorpore la captura final de la pantalla con los resultados alcanzados.

EVALUACIÓN DE LA ACTIVIDAD:

Para la autoevaluación de la actividad, puede apoyarse en dos tipos de materiales: la lista de chequeo que le ofrecemos a continuación que le puede ayudar para saber si ha incorporado todos los diferentes elementos, y la rúbrica para conocer el nivel de profundización que ha

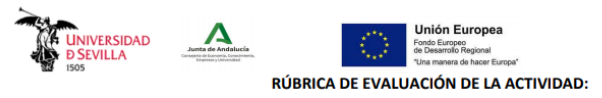
RÚBRICA DE EVALUACIÓN DE LA ACTIVIDAD:

\begin{tabular}{|c|c|c|c|}
\hline & \multicolumn{3}{|c|}{ ESCALA DE VALORACIÓN } \\
\hline \begin{tabular}{|ll}
$\begin{array}{l}\text { CRITERIOS } \\
\text { VALORACIÓN }\end{array}$ & DE \\
\end{tabular} & 1 & 2 & 3 \\
\hline 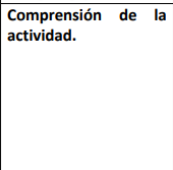 & $\begin{array}{l}\text { No se comprende la } \\
\text { actividad planteada. } \\
\text { No se realiza. }\end{array}$ & $\begin{array}{lr}\text { Demuestra } & \text { poca } \\
\text { comprensión de } & \text { la } \\
\text { actividad. Muchos de } \\
\text { los requerimientos de } \\
\text { la tarea faltan en la } \\
\text { producción } \\
\text { respuesta. }\end{array}$ & $\begin{array}{l}\text { Demuestra } \\
\text { comprensión ， } \\
\text { actividad. La } \\
\text { cantidad } \\
\text { requerimientos } \\
\text { tarea } \\
\text { comprendidas } \\
\text { respuesta ofrecii }\end{array}$ \\
\hline $\begin{array}{l}\text { Claridad de la } \\
\text { respuesta ofrecida }\end{array}$ & $\begin{array}{l}\text { No satisface } \\
\text { prácticamente nada } \\
\text { de los requerimientos } \\
\text { de desempeño. No } \\
\text { aplica los contendidos } \\
\text { presentados para la } \\
\text { realización de la tarea } \\
\text { y no identifica ni los } \\
\text { programas, ni los } \\
\text { localiza en la sopa de } \\
\text { letras, por lo que }\end{array}$ & $\begin{array}{l}\text { Satisface parcialmente } \\
\text { los requerimientos de } \\
\text { desempeño. Localiza } \\
\text { algunos de los } \\
\text { programas rat ratiliza } \\
\text { identificar, y utiliza de } \\
\text { manera ocasional, la } \\
\text { opción de mostrar } \\
\text { ayuda. }\end{array}$ & $\begin{array}{l}\text { Satisface } \\
\text { requerimientos } \\
\text { desempeño. L } \\
\text { la mayoría d } \\
\text { programas } \\
\text { identificar, y } \\
\text { pocas veces, la । } \\
\text { de mostrar } \\
\text { Además, realii } \\
\text { captura de panti } \\
\text { problema algunc }\end{array}$ \\
\hline
\end{tabular}

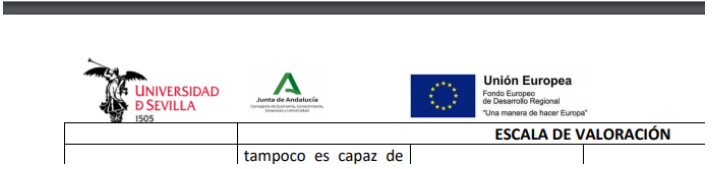

Figura 7. Ejemplo de presentación de tarea.

Fuente: Elaboración propia.

Se debe señalar que, las e-actividades (tareas) propuestas son de diversa tipología: realización de mapas conceptuales, participación en foros, construcción de blog, creación de PLE con determinadas herramientas, organización de actividades para los estudiantes

Evaluación de t-MOOC sobre competencias digitales docentes universitario mediante el juicio de expertos. Selección mediante el coeficiente de competencia experta. Cabero-Almenara, BarrosoOsuna, Palacios-Rodríguez, y Llorente-Cejudo.

Página 10 de 30 
y compañeros, creación de comunidades de aprendizaje.

Por lo que se refiere a los recursos utilizados en los módulos de aprendizaje, apuntar que han sido los siguientes: animaciones didácticas, grabaciones Polimedia (Cabero, 2018), clip de vídeos, infografías, direcciones web y documentos complementarios (PDF).

También se han utilizado diferentes tipos de foros: foros de dudas generales del funcionamiento del t-MOOC, foro de dudas de cada área competencial y foros específicos de actividades.

En definitiva, el t-MOOC cuenta con:

- 66 módulos de aprendizaje (3 por cada competencia DigCompEdu: inicial, intermedio y avanzado).

- 230 tareas repartidas en los módulos de aprendizaje.

- 1 animación con las instrucciones de navegación y uso del t-MOOC.

- 1 animación general (DigCompEdu).

- 6 animaciones específicas de cada área competencial DigCompEdu.

- 22 animaciones específicas de cada competencia DigCompEdu.

- 16 animaciones integradas en los distintos módulos de aprendizaje.

- 24 infografías integradas en los distintos módulos de aprendizaje.

- 11 polimedias integrados en los distintos módulos de aprendizaje.

Finalmente, para la producción del t-MOOC se utilizan una gran diversidad de programas. En concreto: ExeLearning para los módulos de aprendizaje), VYOND para la realización de las animaciones didácticas, Genially para la producción de infografías, Photoshop para el diseño gráfico, Adobe Premiere para la edición de vídeo y Audacity para la ecualización de los audios.

\section{Juicio de expertos}

Para su evaluación, se utiliza la técnica del juicio de expertos, una de las estrategias de evaluación de tecnologías más utilizadas (Barroso et al., 2015). Como señalan Cabero \& Llorente (2013, p.14), "consiste, básicamente, en solicitar a una serie de personas la demanda de un juicio hacia un objeto, un instrumento, un material de enseñanza, o su opinión respecto a un aspecto concreto".

Esta estrategia se está popularizando en la investigación...evaluación educativa y ha sido utilizada para resolver diferentes problemáticas educativas, desde la evaluación de cuestionario hasta la evaluación de recursos tecnológicos (Pérez Juste, 2006; Robles \& Rojas, 2015; Galicia et al., 2017; Peraza et al., 2019; Cabero-Almenara et al., 2020d). De forma paralela, esta estrategia está asociada a los estudios Delphi (López Gómez, 2018).

En su aplicación surgen una serie de problemas entre los que aparecen: su selección y el que una persona puede ser expertas en aspectos específicos y no en otros. Para resolver estos problemas, una de las estrategias que se utiliza para la

Evaluación de t-MOOC sobre competencias digitales docentes universitario mediante el juicio de expertos. Selección mediante el coeficiente de competencia experta. Cabero-Almenara, BarrosoOsuna, Palacios-Rodríguez, y Llorente-Cejudo. 
selección de expertos es el denominado Coeficiente de Competencia Experta (CCE) (Cabero \& Barroso, 2013; Cabero \& Infante, 2014; Martínez \& López Gómez, 2018; Martínez et al., 2018; Nadina et al., 2018). Recientemente, Cruz y Martínez (2020) han realizado una revisión de investigaciones donde se ha utilizado el CCE para la selección de los expertos.

En el presente estudio se establecen dos mecanismos para la identificación de los expertos: en primer lugar, se selección teniendo en cuenta el cumplimiento de dos o más de los siguientes criterios:

- Impartir docencia en Universidades en las asignaturas de "Tecnología Educativa", "Nuevas Tecnologías aplicadas a la Educación”, "Tecnologías de la Información y Comunicación Aplicadas a la Educación" o similares.

- Tener experiencia en el terreno de la formación del profesorado en TIC.

- Haber publicado algún artículo sobre e-learning, formación virtual, blearning, y MOOC, en los últimos cinco años.

- Ser de distintas Universidades Españolas y/o Latinoamericanas.

Uno de los problemas asociados al juicio de experto se refiere al número de expertos que se necesitan para la aplicación. Las propuestas oscilan entre: 15-20 (Malla \& Zabala, 1978), 15-35 (Landeta, 2002); o 15-25 (Witkin \& Altschuld, 1995). Como se señala, se número viene determinado por diferentes aspectos: disponer de diferentes expertos hacia la temática analizada, evitar el menor número de pérdida de sujetos si se consideran diferentes vueltas, el volumen de trabajo que seamos capaces de analizar, la facilidad con que podamos acceder a la información y la rapidez con que debamos ofrecer los resultados preliminares (Cabero \& Barroso, 2013). En este caso, puesto que no existen problemas para trabajar con una amplia base de datos y solamente se realiza una vuelta, se adopta la decisión de trabajar con el mayor número posible.

\section{Procedimiento}

El número de correos electrónicos que se enviaron de acuerdo con los criterios inicialmente tenidos en cuenta es de 369. De ellos, tras las dos semanas en las cuales el cuestionario se mantiene abierto, se reciben 172 contestaciones.

Debido al interés de afinar en el proceso de selección de los expertos finales, se aplica el CCE (Cabero \& Barroso, 2013; Cabero \& Infante, 2014; López Gómez, 2018; Martínez \& otros, 2018). Dicho índice se obtiene a partir de la autopercepción que el experto tiene sobre su nivel de conocimiento respecto a la temática analizada, así como de las fuentes que le permiten argumentar la decisión adoptada.

Para su obtención, se utiliza la fórmula: $K=1 / 2(K c+K a)$. Donde $K c$ es el "coeficiente de conocimiento", y se obtiene de la puntuación ofrecida directamente por el experto en la siguiente pregunta:

Marque en la casilla que le corresponde el grado de conocimiento que usted posee acerca de temáticas como las siguientes: formación del profesorado en TIC, competencias digitales, alfabetización digital.... Valórese en una escala de 0 a 10 (considerando el 0 como no tener absolutamente ningún conocimiento y 10 de pleno

Evaluación de t-MOOC sobre competencias digitales docentes universitario mediante el juicio de expertos. Selección mediante el coeficiente de competencia experta. Cabero-Almenara, BarrosoOsuna, Palacios-Rodríguez, y Llorente-Cejudo. 
conocimiento del estado de la cuestión.

Ka es el coeficiente de argumentación, que se alcanzan sumando las opciones especificadas por el experto en la tabla que cumplimenta de la siguiente pregunta:

Valore el grado de influencia que cada una de las fuentes que le presentamos a continuación, ha tenido en su conocimiento y criterios sobre el tema de la formación del profesorado en TIC, competencias digitales, ..., alfabetización digital.

Tabla 2. Indicadores y valores relacionados de Ka.

\begin{tabular}{lccc}
\hline & BAJO & MEDIO & ALTO \\
\hline Análisis teóricos realizado por usted & 0,10 & 0,20 & 0,30 \\
Su experiencia obtenida de su actividad práctica & 0,20 & 0,40 & 0,50 \\
Estudio de trabajo sobre el tema, de autores españoles. & 0,05 & 0,05 & 0,05 \\
Estudio de trabajo sobre el tema, de autores extranjeros. & 0,05 & 0,05 & 0,05 \\
Su propio conocimiento acerca del estado del problema en el extranjero & 0,05 & 0,05 & 0,05 \\
Su intuición sobre el tema abordado. & 0,05 & 0,05 & 0,05 \\
\hline
\end{tabular}

Fuente: Elaboración propia.

Los valores que se utilizan para determinar la posición del experto son:

- $0,8<K<1,0$ coeficiente de competencia alto

- $0,5<\mathrm{K}<0,8$ coeficiente de competencia medio

- $\mathrm{K}<0,5$ coeficiente de competencia bajo

El número de expertos a los que inicialmente se les envió el cuestionario fue de 369. En este caso, para afinar el proceso de selección de expertos, se adopta que tuvieran un valor 0,9 o superior. Esto permite identificar 172 expertos; lo que suponía el 52,76\% del total de respuestas obtenidas.

A continuación, se presentan algunas de las características de estos expertos con valor CCE $\geq 0.9$.

Tabla 3. Algunas características de los expertos respecto a la titulación, institución donde trabaja y actividad profesional.

\begin{tabular}{lccc}
\hline Variable & Niveles & $\mathbf{f}$ & $\%$ \\
\hline Titulación & Grado/Licenciado & 2 & 1,2 \\
& Máster & 30 & 17,4 \\
& Doctorado & 140 & 81,4 \\
Institución donde trabaja: Centro Universitario & $\mathrm{Si}$ & 170 & 98,8 \\
& $\mathrm{No}$ & 2 & 1,2 \\
Institución donde trabaja: Centro no Universitario & $\mathrm{Si}$ & 24 & 14,0 \\
\hline
\end{tabular}

Evaluación de t-MOOC sobre competencias digitales docentes universitario mediante el juicio de expertos. Selección mediante el coeficiente de competencia experta. Cabero-Almenara, BarrosoOsuna, Palacios-Rodríguez, y Llorente-Cejudo. 
RED. Revista de Educación a Distancia. Núm. 67, Vol. 21. Artíc. 2, 30-Sep-2021

DOI: http://dx.doi.org/10.6018/red.476891

\begin{tabular}{lcccc}
\hline Variable & Niveles & $\mathbf{f}$ & $\%$ \\
\hline & $\mathrm{No}$ & 148 & 86,0 \\
Institución donde trabaja: Empresa relacionada con formación & $\mathrm{Si}$ & 38 & 22,1 \\
& $\mathrm{No}$ & 134 & 77,9 \\
Institución donde trabaja: Empresa relacionada con la & $\mathrm{Si}$ & 16 & 9,3 \\
producción de tecnología & $\mathrm{No}$ & 156 & 90,7 \\
Actividad básica profesional: docente & $\mathrm{Si}$ & 170 & 98,8 \\
& $\mathrm{No}$ & 2 & 1,2 \\
Actividad básica profesional: Técnica & $\mathrm{Si}$ & 38 & 22,1 \\
& $\mathrm{No}$ & 134 & 77,9 \\
Actividad básica profesional: Gestión & $\mathrm{Si}$ & 98 & 57,0 \\
& $\mathrm{No}$ & 74 & 43,0 \\
Actividad básica profesional: Investigadora & $\mathrm{Si}$ & 164 & 95,3 \\
& $\mathrm{No}$ & 8 & 4,7 \\
\hline
\end{tabular}

Fuente: Elaboración propia.

También se pregunta por una serie de cuestiones relacionadas con su ámbito de trabajo, presentadas en la Tabla 4.

Tabla 4. Características de los jueces seleccionados a partir de CCE $\geq 0,9$.

\begin{tabular}{|c|c|c|c|}
\hline Variable & $\mathbf{R}$ & $f$ & $\%$ \\
\hline $\begin{array}{l}\text { ¿Ha impartido a lo largo de su vida profesional } \\
\text { asignatura/contenidos/materias/acciones formativas relacionadas con el terreno } \\
\text { de la Tecnología Educativa, Nuevas Tecnologías Aplicadas a la Educación, } \\
\text { Formación a distancia, formación virtual, TIC aplicadas a la educación, } \\
\text { ¿formación del profesorado en TIC? }\end{array}$ & $\begin{array}{l}\mathrm{Si} \\
\text { No }\end{array}$ & $\begin{array}{l}168 \\
4\end{array}$ & $\begin{array}{l}97,7 \\
2,3\end{array}$ \\
\hline $\begin{array}{l}\text { ¿Ha dirigido o participado en alguna investigación relacionada con aspectos de } \\
\text { la formación virtual, formación a distancia, e-learning, formación del profesorado } \\
\text { en TIC, competencias digitales de los docentes y estudiantes, alfabetización } \\
\text { digital...? }\end{array}$ & $\begin{array}{l}\mathrm{Si} \\
\text { No }\end{array}$ & $\begin{array}{l}170 \\
2\end{array}$ & $\begin{array}{c}98,8 \\
1,2\end{array}$ \\
\hline $\begin{array}{l}\text { ¿Ha realizado o participado en alguna publicación relacionada con aspectos de } \\
\text { la formación virtual, formación a distancia, e-learning, formación del profesorado } \\
\text { en TIC, competencias digitales de los docentes y estudiantes, alfabetización } \\
\text { digital, ...? }\end{array}$ & $\begin{array}{l}\mathrm{Si} \\
\text { No }\end{array}$ & $\begin{array}{l}166 \\
6\end{array}$ & $\begin{array}{l}96,5 \\
3,5\end{array}$ \\
\hline $\begin{array}{l}\text { ¿Ha impartido a lo largo de su vida profesional } \\
\text { asignatura/contenidos/materias/charlas/conferencias relacionadas con la } \\
\text { formación del profesorado en TIC, competencias digitales, alfabetización } \\
\text { digital...? }\end{array}$ & Si & $\begin{array}{l}170 \\
2\end{array}$ & $\begin{array}{l}98,8 \\
1,2\end{array}$ \\
\hline
\end{tabular}

Fuente: Elaboración propia

Evaluación de t-MOOC sobre competencias digitales docentes universitario mediante el juicio de expertos. Selección mediante el coeficiente de competencia experta. Cabero-Almenara, BarrosoOsuna, Palacios-Rodríguez, y Llorente-Cejudo. 
Como se observa, la gran mayoría de los expertos identificados señalan que tenían experiencia de docencia, publicaciones e investigaciones en temática de las TIC yla competencia y alfabetización digital del profesorado.

El instrumento de recogida de información contiene dos grandes apartados. En el primero, se acopia información respecto algunas características del experto (titulación, actividad profesional, lugar en el que trabaja, ...) y se incorporan las preguntas destinadas a elaborar el CCE; y en el segundo, se le solicitaba que valorara el t-MOOC. Para ello, se realiza una adaptación del cuestionario elaborado por Cabero \& Llorente (2015), utilizado para la evaluación del diseño de otras tecnologías. Al final, se realiza una pregunta abierta para realizar y obtener propuestas específicas de modificación y mejora.

El cuestionario se administra vía internet, y se realiza con la herramienta Google Forms: https://cutt.ly/PzZsfCV. También se debe indicar que dicho cuestionario incorpora un clip de vídeo donde se explicaba el funcionamiento del t-MOOC (Figura 8).
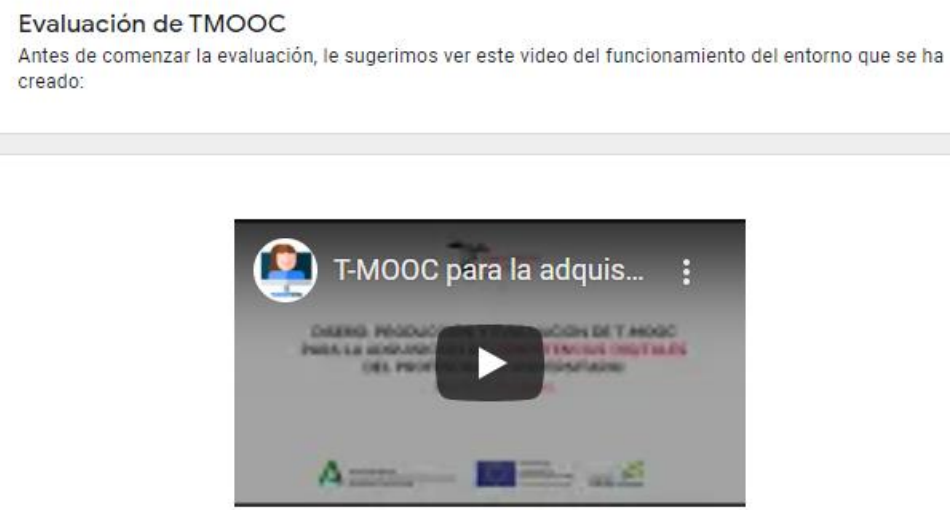

Para entrar en el entorno, debes usar esta dirección web: https://www.tmoocuni.es

-Contraseña: Evaluacion21? (respetar mayúscula e interrogación)

Figura 8. Pantalla del cuestionario de evaluación del t-MOOC.

Fuente: Elaboración propia.

La recogida de información se lleva a cabo entre los meses de noviembrediciembre del 2020.

El instrumento utilizado usa un escalamiento de tipo Likert, con seis opciones de respuestas: 1. MN= Muy negativo / Muy en desacuerdo / Muy difícil; 2. N= Negativo /En desacuerdo / Difícil; 3. R-= Regular negativo / Moderadamente en desacuerdo / Moderadamente difícil; 4. $\mathrm{R}+=$ Regular positivo / Moderadamente de acuerdo /

Evaluación de t-MOOC sobre competencias digitales docentes universitario mediante el juicio de expertos. Selección mediante el coeficiente de competencia experta. Cabero-Almenara, BarrosoOsuna, Palacios-Rodríguez, y Llorente-Cejudo. 
Moderadamente fácil; 5. P= Positivo / De acuerdo / Fácil; y 6. MP= Muy positivo / Muy de acuerdo / Muy fácil. Las dimensiones analizadas son: aspectos técnicos, facilidad de uso, diversidad de recursos y actividades y calidad del contenido.

\section{RESULTADOS}

Inicialmente, se presentan los valores medios y las desviaciones típicas alcanzadas en las cuatro grandes dimensiones que constituyen el instrumento de recogida de información, además de la valoración global realizada del t-MOOC.

Tabla 4. Valoración media y desviación típica realizada por los expertos en los entornos percibidos de forma conjunta y separada.

\begin{tabular}{lcc}
\hline Dimensiones & M. & D.T. \\
\hline Aspectos técnicos & 5,24 & 0,69 \\
Facilidad de uso & 5,20 & 0,76 \\
Diversidad de recursos y actividades & 5,26 & 0,80 \\
Calidad de los contenidos & 5,46 & 0,73 \\
Total & 5,29 & 0,67 \\
\hline
\end{tabular}

Fuente: Elaboración propia.

Las puntuaciones medias alcanzadas permiten señalar que, tanto en la valoración de cada dimensión como de forma global, los expertos han valorado el t-MOOC de manera muy positiva. Al mismo tiempo, se puede indicar que los valores bajos de desviaciones típicas muestran la coexistencia de las respuestas ofrecidas por los expertos en las respuestas ofrecidas.

A continuación, se ofrecen las puntuaciones alcanzadas en los diferentes ítems que conforman cada una de las dimensiones. En la Tabla 5 se presentan los valores medios y las desviaciones típicas obtenidas en la dimensión "aspectos técnicos y estéticos".

Tabla 5. Valoración media y desviación típica realizada por los expertos en los ítems de la dimensión "aspectos técnicos y estéticos."

\begin{tabular}{lcc}
\hline Aspectos técnicos y estéticos & Media & D. tp \\
\hline El funcionamiento del t-MOOC que te hemos presentado es: & 5,37 & 0,715 \\
En general, la estética del t-MOOC producido la consideras: & 5,03 & 0,978 \\
En general, el funcionamiento técnico del t-MOOC producido lo calificarías de: & 5,39 & 0,746 \\
En general, ¿Cómo valorarías la presentación de la información en la pantalla? & 5,13 & 0,873
\end{tabular}

Fuente: Elaboración propia.

Por lo que se refiere a los aspectos técnicos y estéticos, las valoraciones de los

Evaluación de t-MOOC sobre competencias digitales docentes universitario mediante el juicio de expertos. Selección mediante el coeficiente de competencia experta. Cabero-Almenara, BarrosoOsuna, Palacios-Rodríguez, y Llorente-Cejudo.

Página 16 de 30 
expertos permiten señalar dos aspectos fundamentales: por una parte, el funcionamiento de los diferentes elementos es correcto y adecuado, por otra parte, desde el punto de vista estético el material producido se valora positivamente y puede considerarse de atractivo. En ninguna de las preguntas se observa una puntuación inferior al 5,20, lo que sugiere una alta valoración dentro de la escala ofrecida.

La siguiente dimensión se refiere a la "facilidad de utilización" del entorno creado por el usuario. En la Tabla 6 se presentan los valores medios y las desviaciones típicas alcanzadas en cada uno de los ítems.

Tabla 6. Valoración de los expertos sobre los ítems de la dimensión "facilidad de utilización."

\begin{tabular}{|c|c|c|}
\hline Facilidad de utilización & Media & D. tp. \\
\hline $\begin{array}{l}\text { ¿Cómo calificaría la facilidad de uso y manejo del t-MOOC que te hemos } \\
\text { presentado? }\end{array}$ & 5,34 & 0,799 \\
\hline $\begin{array}{l}\text { ¿Cómo calificarías la facilidad de comprensión del funcionamiento técnico del t- } \\
\text { MOOC que te hemos presentado? }\end{array}$ & 5,32 & 0,822 \\
\hline $\begin{array}{l}\text { Desde tu punto de vista, ¿Cómo valorarías el diseño general del t-MOOC que } \\
\text { hemos elaborado? }\end{array}$ & 5,14 & 0,868 \\
\hline $\begin{array}{l}\text { Desde tu punto de vista, ¿Cómo valorarías la accesibilidad/usabilidad del t-MOOC } \\
\text { que te hemos presentado? }\end{array}$ & 5,27 & 0,841 \\
\hline $\begin{array}{l}\text { Desde tu punto de vista, ¿Cómo valorarías la flexibilidad de utilización del t-MOOC } \\
\text { que te hemos presentado? }\end{array}$ & 5,26 & 0,823 \\
\hline El utilizar el t-MOOC producido te fue divertido. & 4,70 & 1,165 \\
\hline
\end{tabular}

Fuente: Elaboración propia.

Lo primero a señalar son las altas puntuaciones alcanzadas en cada uno de los ítems, si bien se debe resaltar que en esta dimensión se encuentra el ítem que obtuvo menor valoración media; en concreto, el denominado "El utilizar el t-MOOC producido te fue divertido", ya que fue el único ítem de cuestionario que bajó de la puntuación media de 5 (4.70). En contrapartida, el entorno fue valorado muy positivamente, con puntuaciones superiores al 5,30 en los ítems referidos a la facilidad de uso (“¿Cómo calificaría la facilidad de uso y manejo del t-MOOC que te hemos presentado?" y “¿Cómo calificarías la facilidad de comprensión del funcionamiento técnico del t-MOOC que te hemos presentado?"). Al mismo tiempo y, cercana a la valoración citada anteriormente, se presenta la valoración del ítem “¿Cómo valorarías la accesibilidad/usabilidad del t-MOOC que te hemos presentado?”, que fue de 5,27.

Respecto a la valoración que se realiza de la dimensión "diversidad de recursos y actividades", las puntuaciones medias alcanzadas se presentan en la Tabla 7.

Tabla 7. Valoración de los expertos en lo que se refiere a la dimensión "Diversidad de recursos y actividades."

Evaluación de t-MOOC sobre competencias digitales docentes universitario mediante el juicio de expertos. Selección mediante el coeficiente de competencia experta. Cabero-Almenara, BarrosoOsuna, Palacios-Rodríguez, y Llorente-Cejudo.

Página 17 de 30 
RED. Revista de Educación a Distancia. Núm. 67, Vol. 21. Artíc. 2, 30-Sep-2021

DOI: http://dx.doi.org/10.6018/red.476891

\begin{tabular}{lcc}
\hline Diversidad de recursos y actividades & Media & D. tp. \\
\hline $\begin{array}{l}\text { La diversidad de recursos utilizados en el t-MOOC facilita la comprensión de los } \\
\text { contenidos. }\end{array}$ & 5,17 & 0,927 \\
$\begin{array}{l}\text { Los materiales, lecturas, animaciones, vídeos... ofrecidos en el t-MOOC son claros } \\
\text { y adecuados. }\end{array}$ & 5,29 & 0,917 \\
$\begin{array}{l}\text { La estructura y los materiales del t-MOOC son motivantes para el estudio. } \\
\text { Las actividades ofrecidas en el t-MOOC resultan atractivas e innovadoras. }\end{array}$ & $5,09,946$ \\
$\begin{array}{l}\text { Existen diferentes modalidades y tipos de actividades: de refuerzo, de apoyo, de } \\
\text { ampliación... presentadas en el t-MOOC. }\end{array}$ & 5,25 & $0,9,920$ \\
\hline
\end{tabular}

Fuente: Elaboración propia.

De nuevo, en todos los casos las puntuaciones medias alcanzadas superan la valoración media de 5, sobresaliendo en la dimensión la puntuación otorgada a los ítems: "Los materiales, lecturas, animaciones, vídeos... ofrecidos en el t-MOOC son claros y adecuados" $(5,29)$, y "Existen diferentes modalidades y tipos de actividades: de refuerzo, de apoyo, de ampliación... presentadas en el t-MOOC" la puntuación alcanzada fue de 5,25. Por otro lado, y con la misma puntuación, destacan ítems como "La diversidad de recursos utilizados en el t-MOOC facilita la comprensión de los contenidos" $(5,17)$ y "Las actividades ofrecidas en el t-MOOC resultan atractivas e innovadoras" $(5,17)$.

La última dimensión del cuestionario se refiere a la "calidad de los contenidos". En la Tabla 8, se presentan las medias alcanzadas.

Evaluación de t-MOOC sobre competencias digitales docentes universitario mediante el juicio de expertos. Selección mediante el coeficiente de competencia experta. Cabero-Almenara, BarrosoOsuna, Palacios-Rodríguez, y Llorente-Cejudo.

Página 18 de 30 
RED. Revista de Educación a Distancia. Núm. 67, Vol. 21. Artíc. 2, 30-Sep-2021 DOI: http://dx.doi.org/10.6018/red.476891

Tabla 6. Valoración de los expertos en lo que se refiere a la dimensión "Calidad de los contendios."

\begin{tabular}{lcc}
\hline Calidad de los contenidos & Media & D. tp. \\
\hline Los contenidos del t-MOOC así como su estructura son claros y adecuados. & 5,40 & 0,810 \\
$\begin{array}{l}\text { Los contenidos presentados en el t-MOOC se adecuan a las competencias que se } \\
\text { desean desarrollar. }\end{array}$ & 5,44 & 0,752 \\
Los contenidos del t-MOOC son fáciles de comprender. & 5,40 & 0,700 \\
\hline
\end{tabular}

Fuente: Elaboración propia.

Hay que señalar que en esta dimensión se encuentran los tres ítems que han alcanzado las valoraciones más altas de todo el cuestionario, situándose en una puntuación de 5,40 o superior. Al mismo tiempo, también es donde las puntuaciones de las desviaciones típicas son las menores, lo cual sugiere la igualdad de las valoraciones ofrecidas por los expertos que valoraron el t-MOOC.

A nivel de síntesis, se ofrecen los diez ítems que ofrecieron las puntuaciones más elevadas:

1.- Los contenidos presentados en el t-MOOC se adecuan a las competencias que se desean desarrollar. (5.44).

2.- Los contenidos del t-MOOC, así como su estructura son claros y adecuados. (5.40).

3.- Los contenidos del t-MOOC son fáciles de comprender. (5.40).

4.- En general, el funcionamiento técnico del t-MOOC producido lo calificarías de (5.37).

5.- El funcionamiento del t-MOOC que te hemos presentado es (5.39).

6.- ¿Cómo calificaría la facilidad de uso y manejo del t-MOOC que te hemos presentado? (5.34).

7.- ¿Cómo calificarías la facilidad de comprensión del funcionamiento técnico del tMOOC que te hemos presentado? (5.32).

8.- Los materiales, lecturas, animaciones, vídeos... ofrecidos en le t-MOOC son claros y adecuados (5.29).

9.- Desde tu punto de vista, ¿Cómo valorarías la accesibilidad/usabilidad del t-MOOC que te hemos presentado? (5.27).

10.- Existen diferentes modalidades y tipos de actividades: de refuerzo, de apoyo, de ampliación... presentadas en el t-MOOC (5.25).

En este estudio también se pretende saber si la titulación máxima del experto influye en la valoración realizada En concreto, se formulan las siguientes hipótesis:

H0 (Hipótesis nula): no existen diferencias estadísticamente significativas en las valoraciones realizadas del t-MOOC por los expertos en función de su titulación.

Evaluación de t-MOOC sobre competencias digitales docentes universitario mediante el juicio de expertos. Selección mediante el coeficiente de competencia experta. Cabero-Almenara, BarrosoOsuna, Palacios-Rodríguez, y Llorente-Cejudo.

Página 19 de 30 
RED. Revista de Educación a Distancia. Núm. 67, Vol. 21. Artíc. 2, 30-Sep-2021

DOI: http://dx.doi.org/10.6018/red.476891

H1(Hipótesis alternativa): existen diferencias estadísticamente significativas en las valoraciones realizadas del t-MOOC por los expertos en función de su titulación.

Para ello, se aplica el estadístico no paramétrico $\mathrm{H}$ de Kruskal-Wallis, que permite conocer si existen diferencias estadísticamente significativas entre $N$ muestras independientes (Bisquerra \& Alzina, 2004). Los resultados se presentan en la Tabla 9, a través de las grandes dimensiones que conformaban el cuestionario, así como a la puntuación global alcanzada.

Tabla 9. Resultados prueba $\mathrm{H}$ de Kruskal-Wallis.

\begin{tabular}{lcc}
\hline Dimensión & H de Kruskal-Wallis & Sig. \\
\hline Aspectos técnicos y estéticos & 3,086 &, 214 \\
Facilidad de utilización & 3,114 &, 211 \\
Diversidad de recursos y actividades & 1,325 &, 516 \\
La calidad de los contenidos & 6,754 &, 034 \\
GENERAL & 4,053 &, 132 \\
\hline
\end{tabular}

Fuente: Elaboración propia.

Los resultados permiten rechazar la $\mathrm{H} 0$ en la dimensión "calidad de los contenidos", a un nivel de significación de $p \leq .05$. Se puede concluir que, salvo en la dimensión "calidad de los contenidos", no existen diferencias significativas en las valoraciones que realizaron los expertos en función de su titulación para la globalidad del instrumento y sus dimensiones "aspectos técnicos y estéticos", "facilidad de utilización" y "diversidad de recursos y actividades".

Con el objeto de conocer entre que colectivos se daban las diferencias en la dimensión "calidad de los contenidos", que es donde se rechazó la H0, se aplica la prueba posthoc de ANOVA para el test de Kruskal Wallis (test de Dunn). Esta prueba es la más indicada para conocer si existen diferencias estadísticamente significativas por parejas entre los grupos establecidos en Kruskal Wallis (Bisquerra \& Alzina, 2004). En las Tabla 10 se presentan los valores alcanzados.

Tabla 10. Resultados test de Dunn.

\begin{tabular}{lcccc}
\hline & $\begin{array}{c}\text { Estadístico de } \\
\text { prueba }\end{array}$ & Desv. Error & $\begin{array}{c}\text { Desv. Estadístico de } \\
\text { prueba }\end{array}$ & Sig. \\
\hline Grado-Máster & $-59,200$ & 34,230 & $-1,729$ &, 084 \\
Grado-Doctorado & $-73,314$ & 33,379 & $-2,196$ &, 028 \\
Máster-Doctorado & $-14,114$ & 9,430 & $-1,497$ &, 134 \\
\hline
\end{tabular}

Evaluación de t-MOOC sobre competencias digitales docentes universitario mediante el juicio de expertos. Selección mediante el coeficiente de competencia experta. Cabero-Almenara, BarrosoOsuna, Palacios-Rodríguez, y Llorente-Cejudo.

Página 20 de 30 
RED. Revista de Educación a Distancia. Núm. 67, Vol. 21. Artíc. 2, 30-Sep-2021 DOI: http://dx.doi.org/10.6018/red.476891

Los valores encontrados llevan a señalar que las diferencias significativas se han dado entre los jueces cuya máxima titulación era poseer el grado y aquellos que poseían el título de doctor. A continuación, se analizan los rangos promedio para saber qué grupo otorga mayores valores de puntuación (Tabla 11).

Tabla 11. Resultados rango promedio prueba $\mathrm{H}$ de Kruskal-Wallis.

\begin{tabular}{llc}
\hline & Titulación académica máxima que posee & Rango promedio \\
\hline $\begin{array}{l}\text { La_calidad_de_los } \\
\text { contenidos }\end{array}$ & Grado & 16,50 \\
& & \\
& Máster & 75,70 \\
& Doctorado & 89,81 \\
\hline
\end{tabular}

Tal y como se puede comprobar, los expertos con el título de doctor son los que otorgan puntuaciones más altas la calidad de los contenidos del t-MOOC.

De forma paralela, se pretende saber si existen diferencias estadísticamente significativas entre las puntuaciones de los expertos que trabajan o no en una empresa relacionada con la formación. Para ello, se formulan las siguientes hipótesis:

HO (Hipótesis nula): no existen diferencias estadísticamente significativas en las valoraciones realizadas del t-MOOC por los expertos en función de si trabajan o no en una empresa relacionada con la formación.

H1(Hipótesis alternativa): existen diferencias estadísticamente significativas en las valoraciones realizadas del t-MOOC por los expertos en función de si trabajan o no en una empresa relacionada con la formación.

Para ello, se aplica el estadístico U de Mann-Whitney, que permite conocer si existen diferencias estadísticamente significativas entre 2 muestras independientes (Bisquerra \& Alzina, 2004). Los resultados se presentan en la Tabla 12.

Tabla 12. Resultados prueba U de Mann-Whitney

\begin{tabular}{ccc}
\hline & $\begin{array}{c}\text { U de } \\
\text { Mann- } \\
\text { Whitney }\end{array}$ & $\begin{array}{c}\text { Sig. } \\
\text { asintótica(bilateral) }\end{array}$ \\
\hline D1.Aspectos_técnicos_y_estéticos & 1826 & 0,007 \\
D2.Facilidad_de_utilización & 1836 & 0,008 \\
D3.Diversidad_de_recursos_y_actividades & 1606 & 0,000 \\
D4.La_calidad_de_los_contenidos & 1714 & 0,001 \\
GENERAL & 1564 & 0,000 \\
\hline
\end{tabular}

Fuente: Elaboración propia.

Evaluación de t-MOOC sobre competencias digitales docentes universitario mediante el juicio de expertos. Selección mediante el coeficiente de competencia experta. Cabero-Almenara, BarrosoOsuna, Palacios-Rodríguez, y Llorente-Cejudo. 
RED. Revista de Educación a Distancia. Núm. 67, Vol. 21. Artíc. 2, 30-Sep-2021

DOI: http://dx.doi.org/10.6018/red.476891

Como se puede apreciar, se puede afirmar al 99\% que existen diferencias estadísticamente significativas ( $p \leq .05$ ) entre las puntuaciones otorgadas por los expertos que trabajan en una empresa relacionada con la formación y los que no. Para conocer qué grupo otorga las puntuaciones más altas, se procede a realizar un análisis de rango promedio (Tabla 12).

Tabla 12. Resultados rango promedio prueba $U$ de Mann-Whitney

\begin{tabular}{lcc}
\hline & $\begin{array}{c}\text { Trabaja en una empresa } \\
\text { relacionada con la } \\
\text { formación }\end{array}$ & Rango promedio \\
\hline D1.Aspectos_técnicos_y_estéticos & Sí & 105,45 \\
D2.Facilidad_de_utilización & No & 81,13 \\
& Sí & 105,18 \\
D3.Diversidad_de_recursos_y_actividades & No & 81,2 \\
D4.La_calidad_de_los_contenidos & Sí & 111,24 \\
& No & 79,49 \\
GENERAL & Sí & 108,39 \\
& No & 80,29 \\
\hline
\end{tabular}

Fuente: Elaboración propia.

De acuerdo con los valores obtenidos, el grupo de expertos que trabajan en una empresa relacionada con la formación valoran de forma más positiva todos los aspectos evaluados del t-MOOC: aspectos técnicos y estéticos, facilidad de utilización, diversidad de recursos y actividades, calidad de los contenidos y t-MOOC en general.

Finalmente, se exponen algunos de los comentarios obtenidos por los expertos que han respondido a la pregunta "comentarios adicionales" (Tabla 13). Se ha tratado de clasificarlos según sean aspectos positivos a tener en cuenta y aspectos a mejorar.

Tabla 13. Algunos aspectos positivos y a mejorar según los expertos.

\begin{tabular}{|c|c|}
\hline Aspectos positivos & Aspectos a mejorar \\
\hline Es un entorno amigable y fácil de utilizar & $\begin{array}{c}\text { Quizá incidiría en el atractivo visual, y si fuese } \\
\text { posible, mayor número de actividades participativas } \\
\text { y dinámicas de trabajo en equipo, debate, } \\
\text { retroalimentación y coevaluación de las tareas } \\
\text { planteadas. }\end{array}$ \\
\hline $\begin{array}{c}\text { Valoro positivamente la posibilidad de trabajar por } \\
\text { competencias y por diferentes niveles (inicial, } \\
\text { intermedio y avanzado). }\end{array}$ & $\begin{array}{l}\text { Me hubiera gustado una estructura del curso no tan } \\
\text { lineal, tal vez utilizar el formato de libro u otro diseño } \\
\text { que no haga ver al usuario que el curso "es largo". }\end{array}$ \\
\hline Los vídeos son divertidos y cortos lo que hace & $\begin{array}{l}\text { El curso requiere mejoras más formales que de } \\
\text { contenido. La usabilidad es positiva, pero }\end{array}$ \\
\hline
\end{tabular}

Evaluación de t-MOOC sobre competencias digitales docentes universitario mediante el juicio de expertos. Selección mediante el coeficiente de competencia experta. Cabero-Almenara, BarrosoOsuna, Palacios-Rodríguez, y Llorente-Cejudo.

Página 22 de 30 
RED. Revista de Educación a Distancia. Núm. 67, Vol. 21. Artíc. 2, 30-Sep-2021

DOI: http://dx.doi.org/10.6018/red.476891

atractivo el inicio de cada Área.

Un trabajo muy potente. Un pull de actividades muy claras y bien graduadas. Los materiales de introducción muy bien dimensionaos. Enhorabuena por este trabajo ingente que abre camino para todos.

Es un curso dinámico con buenos recursos digitales de apoyo, la estructura se comprende de forma adecuada.

Me parece una gran estrategia separar por niveles competenciales en t-MOOC. Además, dentro de cada nivel competencial debe haber (infiero) grados de dominio, es decir para obtención de información, para gestión de información y luego Generación y aplicación del Conocimiento. El refuerzo competencial docente es fundamental para poder transmitir y compartir mediante el proceso de Enseñanza-Aprendizaje, al discente.

Me pareció un trabajo organizado, claro y pertinente. Será de gran utilidad para el fin que se desea. formalmente el entorno no resulta atractivo. También es cierto que depende o está condicionado por el perfil o destinatario final de este curso.

Se recomienda que el usuario tenga acceso al índice y al primer bloque y que el resto se haga visible una vez superado el anterior para evitar la sensación de sobrecarga.

Desde mi percepción, al visualizar la totalidad del tMOOC en el formato en que se presenta -un tema debajo del otro- todos con la misma estética y sin muchas diferencias entre uno y otro, resulta difícil ubicarse. Estimo que cuando se tome el curso, como los temas se irán presentando de manera secuencial, esa dificultad sería menor. Extrañé el desarrollo de contenidos. Cada tema inicia con una video animación en el que se hace una breve presentación y luego deriva a actividades. ¿Desde qué marcos conceptuales se van a desarrollar las mismas?, ¿desde los conocimientos previos de los cursantes?, ¿desde el sentido común?, ¿desde sus propias exploraciones en la web sobre el tema? No revisé la totalidad de las actividades, pero aquellas que pude mirar no exigen la reflexión sobre fundamentos pedagógicos acerca de las decisiones que se toman... es decir el para qué o por qué. También se extraña la posibilidad de participar en actividades colaborativas $o$ de intercambio con otros colegas.

Recomendaría colocar más imágenes, iconos que muestren la información de forma más agradable.

Solamente recomendaría revisar las rúbricas de evaluación, tanto en claridad del escrito como en los objetivos o requerimientos de las actividades. Asimismo, revisar las listas de cotejo para la entrega de actividades, algunas de las preguntas no tienen una redacción clara.

Fuente: Elaboración propia.

\section{DISCUSIÓN Y CONCLUSIONES}

Las conclusiones de la investigación van en diferentes direcciones; unas, referidas al procedimiento seguido para la evaluación y para la selección de los evaluadores. Otras, en lo referido a la construcción del t-MOOC.

Respecto a la primera, resaltar que el trabajo presentado permite corroborar la significación del proceso seguido para la selección de los expertos, que constituía dos

Evaluación de t-MOOC sobre competencias digitales docentes universitario mediante el juicio de expertos. Selección mediante el coeficiente de competencia experta. Cabero-Almenara, BarrosoOsuna, Palacios-Rodríguez, y Llorente-Cejudo.

Página 23 de 30 
RED. Revista de Educación a Distancia. Núm. 67, Vol. 21. Artíc. 2, 30-Sep-2021

DOI: http://dx.doi.org/10.6018/red.476891

fases: una selección previa en función de datos biográficos y curriculares de los expertos y una segunda el "Coeficiente de Competencia Experta." La primera establecería una primera selección general, realizada por los propios investigadores, y la segunda, más centrada en el objeto a evaluar, contemplaría la autoevaluación por la persona respecto a su competencia para ello.

Por lo que se refiere al "Coeficiente de Competencia Experta" (Cabero \& Llorente, 2013; Cabero-Almenara, Barroso-Osuna, Palacios-Rodríguez \& LlorenteCejudo, 2020; Cruz \& Martínez, 2020) a la hora de discriminar la experticia de los encargados de la evaluación de los productos realizados en el seno de las investigaciones. Sin embargo, independientemente de la realización de esta prueba, se señala que se hace necesaria la realización de un filtro inicial, como el que se realiza en la presente investigación; es decir, la selección previa por el equipo de investigación y su afinamiento con la autovaloración por parte del experto preguntado.

La eficacia del procedimiento viene también avalada por la significación de las valoraciones del t-MOOC realizada por los expertos, que permitieron mejorar de forma considerable algunos aspectos del t-MOOC. En este sentido, la versión final del t-MOOC incluye una estructura menos lineal, donde las áreas competenciales están claramente diferenciadas mediante elementos multimedia. De la misma forma, se han modificado muchas de las tareas que, a juicio de los expertos, necesitaban ser mejoradas. Finalmente, se ha mejorado la presentación de contenido incluyendo más hiperenlaces y material complementario.

Los resultados avalan también una forma de diseñar el t-MOOC, caracterizada por la utilización de diferentes recursos para la presentación de la información (vídeos, animaciones, infografías, direccionado a sitios web...) y la realización de actividades o tareas en cada módulo por parte del estudiante que sigue el MOOC, para pasar a los siguientes niveles. Esta forma de diseño sugiere la necesidad de pensar en formas de diseño específicos para los materiales utilizados en la formación on-line, que no sea una mera traslación digital de los recursos impresos (Sahasrabudhe \& Kanungo, 2014; Ljbojevic, Vaskovic, Stankovic \& Vaskovic, 2015; Salim \& Luo, 2019, SHFT, 2019) y de incorporar e-actividades a realizar por los estudiantes (Silva, 2017; Burcin, Gemikonakli, Duman \& Kirksekiz, Kiyici, 2020; Cabero-Almenara \& Palacios-Rodríguez, 2021).

Por último, se concluye señalando que esta herramienta permite formar a los profesores universitarios en materia de competencias digitales, dentro del Marco DigCompEdu. Ello permite el abordaje del plan de formación del profesorado desde la perspectiva seleccionada por los jueces, aunque también se debe indicar a las instituciones las directrices para establecer los planes de formación de los docentes en CDD.

Lo comentado lleva sugerir diferentes líneas futuras de investigación, como replicar el estudio para dos o tres vueltas. Ello exigiría una menor utilización de expertos y requeriría un compromiso previo de los mismos a la participación en la investigación en un tiempo mayor. Otra posible línea de investigación es realizar el estudio en otros contextos, como el no universitario.

Evaluación de t-MOOC sobre competencias digitales docentes universitario mediante el juicio de expertos. Selección mediante el coeficiente de competencia experta. Cabero-Almenara, BarrosoOsuna, Palacios-Rodríguez, y Llorente-Cejudo.

Página 24 de 30 
RED. Revista de Educación a Distancia. Núm. 67, Vol. 21. Artíc. 2, 30-Sep-2021

DOI: http://dx.doi.org/10.6018/red.476891

Presentación del artículo: 15 de abril de 2021

Fecha de aprobación: 20 de julio de 2021

Fecha de publicación: 30 de septiembre de 2021

Cabero-Almenara, J., Barroso-Osuna, J., Palacios-Rodríguez, A. \& Llorente-Cejudo, C. (2021). Evaluación de t-MOOC universitario sobre competencias digitales docentes mediante juicio de expertos según el Marco DigCompEdu. RED. Revista Educación a Distancia, 21(67). http://dx.doi.org/10.6018/red.476891

\section{Financiación}

El presente artículo es parte del proyecto Diseño, producción y evaluación de t-Mooc para la adquisición por los docentes de competencias digitales docentes (RTI2018-097214-BC31) financiado por el Ministerio de Ciencia, Innovación y Universidades.

\section{REFERENCIAS}

Aguayo, R. \& Bravo, J. (2017). Implantación de un SPOC en la educación a distancia Para la mejora del proceso de enseñanza-aprendizaje. Revista Tecnología, Ciencia y Educación, 6, 129-142.

Albelbisi, N., Yusop, F. \& Mohd, U. (2018). Mapping the Factors Influencing Success of Massive Open Online Courses (MOOC) in Higher Education. EURASIA Journal of Mathematics, Science and Technology Education, 14(7), 2995-3012.

Amaya, A., Salazar, M., Zúñiga, E. \& Ávila, A. (2018). Empoderar a los profesores en su quehacer académico a través de certificaciones internacionales en competencias digitales. Apertura, 10(1), 104-115. Doi: http://dx.doi.org/10.18381/Ap.v10n1.1174.

Barroso, J., Cabero, J. \& Llorente, M.C. (2015). El diseño, la producción y la evaluación de TIC aplicadas a los procesos de enseñanza-aprendizaje, en Cabero, J. y Barroso, J. (coords.). Nuevos retos en tecnología educativa, Madrid: Síntesis, 69-85.

Benet, A., García, I., Sanahuja, A. \& Nieto, R. (2018). Nuevos horizontes formativos: una experiencia del MOOC como recurso en la formación continua. Apertura, 10(1), 88-103, http://dx.doi.org/10.18381/Ap.v10n1.1151.

Bisquerra, R. \& Alzina, R. B. (2004). Metodología de la investigación educativa. Editorial La Muralla.

Bozkurt, A., Ozdamar, N. \& De Waard, I. (2016). Research Trends in Massive Open Online Course (MOOC). Open Praxis, 8(3), 203-221.

Burcin, N., Gemikonakli, O., Duman, I., Kirksekiz, A. \& Kiyici, M. (2020). Evaluating students experiences using a virtual learning environment: satisfaction and preferences. Education Tech Research Dev., 68, 437-462, https://doi.org/10.1007/s11423-01909705-z.

Evaluación de t-MOOC sobre competencias digitales docentes universitario mediante el juicio de expertos. Selección mediante el coeficiente de competencia experta. Cabero-Almenara, BarrosoOsuna, Palacios-Rodríguez, y Llorente-Cejudo.

Página 25 de 30 
RED. Revista de Educación a Distancia. Núm. 67, Vol. 21. Artíc. 2, 30-Sep-2021

DOI: http://dx.doi.org/10.6018/red.476891

Cabero, J. \& Barroso, J. (2013). La utilización del juicio de experto para la evaluación de TIC: el Coeficiente de competencia experta. Bordón. Revista de Pedagogía, 65(2), 2538 .

Cabero, J. \& Infante, A. (2014). Empleo del método Delphi y su empleo en la investigación en comunicación y educación. Edutec. Revista Electrónica de Tecnología Educativa, 48. Recuperado de http://goo.gl/zDnTm9.

Cabero, J. \& Llorente, M.C. (2013), La aplicación del juicio de experto como técnica de evaluación de las tecnologías de la información (TIC). Eduweb. Revista de Tecnología de Información y Comunicación en Educación, 7(2), 11-22.

Cabero, J. \& Martínez, A. (2019). Las tecnologías de la información y comunicación y la formación inicial de los docentes. Modelos y competencias digitales. Profesorado. Revista de Curriculum y Formación del Profesorado, 23(3), 247-.268.

Cabero, J. (2018). La incorporación de las producciones Polimedias a la formación universitaria. Sevilla: SAV de la Universidad de Sevilla.

Cabero, J. \& Llorente, C. (2015). Entornos Personales de Aprendizaje (PLE): Valoración Educativa a través de Expertos. Areté. Revista Digital del Doctorado en Educación de la Universidad Central de Venezuela. 1 (1), 7 - 19.

Cabero, J. \& otros (2014). Manual para el Desarrollo de la Formación Virtual - Intec Libro de Estilo. Santo Domingo: INTEC.

Cabero, J., Llorente, M.C. \& Vázquez, A. (2014): Las tipologías de los MOOC: su diseño e implicaciones educativas. Profesorado. Revista de Curriculum y formación del profesorado, 18, 1, 13-26.

Cabero-Almenara J, Barroso-Osuna J, Gutiérrez-Castillo J-J, \& Palacios-Rodríguez A. (2021). The Teaching Digital Competence of Health Sciences Teachers. A Study at Andalusian Universities (Spain). International Journal of Environmental Research and Public Health, 18(5), 2552.

Cabero...Almenara, J. \& Palacios...Rodríguez, A. (2020). Marco Europeo de Competencia Digital Docente «DigCompEdu». Traducción y adaptación del cuestionario «DigCompEdu Check $\cdots$ In». EDMETIC, 9(1), 213--234. Doi: https://doi.org/10.21071/edmetic.v9i1.12462.

Cabero-Almenara, J., Barroso-Osuna, J., Palacios-Rodríguez, A. \& Llorente-Cejudo, C. (2020). Marcos de Competencias Digitales para docentes universitarios: su evaluación a través del coeficiente competencia experta. Revista Electrónica Interuniversitaria de Formación del Profesorado, 23(2). https://doi.org/10.6018/reifop.413601.

Cabero-Almenara, J., Barroso-Osuna, J., Rodríguez-Gallego, M. \& Palacios-Rodríguez, A. (2020). La Competencia Digital Docente. El caso de las universidades andaluzas. Aula Abierta, 49(4), 363-372.

Cabero-Almenara, J., Gutiérrez-Castillo, J. J., Palacios-Rodríguez, A. \& Barroso-Osuna, J. (2021). Comparative European DigCompEdu Framework (JRC) and Common Framework for Teaching Digital Competence (INTEF) through expert judgment. Texto Livre: Linguagem E Tecnologia, 14(1), e25740.

Evaluación de t-MOOC sobre competencias digitales docentes universitario mediante el juicio de expertos. Selección mediante el coeficiente de competencia experta. Cabero-Almenara, BarrosoOsuna, Palacios-Rodríguez, y Llorente-Cejudo.

Página 26 de 30 
RED. Revista de Educación a Distancia. Núm. 67, Vol. 21. Artíc. 2, 30-Sep-2021

DOI: http://dx.doi.org/10.6018/red.476891

Cabero-Almenara, J., Gutiérrez-Castillo, J.-J., Palacios-Rodríguez, A. \& Barroso-Osuna, J. (2020). Development of the Teacher Digital Competence Validation of DigCompEdu Check-In Questionnaire in the University Context of Andalusia (Spain). Sustainability 2020, Vol. 12, Page 6094, 12(15), 6094.

Cabero-Almenara, J., Romero-Tena, R. \& Palacios-Rodríguez, A. (2020). Evaluation of Teacher Digital Competence Frameworks Through Expert Judgement: the Use of the Expert Competence Coefficient. Journal of New Approaches in Educational Research, 9(2), 275-293. doi: 10.7821/naer.2020.7.578

Cabero-Almenara, J. \& Palacios-Rodríguez, A. (2021). La evaluación de la educación virtual: las e-actividades. RIED. Revista Iberoamericana de Educación a Distancia, 24(2), (versión preprint). https://doi.org/10.5944/ried.24.2.28994.

Castaño-Muñoz, J., Kalz, M., Kreijns, K. \& Punie, Y. (2018). Who is taking MOOCs for teachers' professional development on the use of ICT? A crosssectional study from Spain. Technology, Pedagogy and Education, 27:5, 607-624, DOI: 10.1080/1475939X.2018.1528997.

Comisión Europea (2017). European Framework for the Digital Competence of Educators

(DigCompEdu). https://ec.europa.eu/jrc/en/digcompedu.

Consejo de la Unión Europea. (2006). Recomendación del Parlamento Europeo y del Consejo de 18 de diciembre de 2006 sobre las competencias clave para el aprendizaje permanente. Bruselas: Diario Oficial de la Unión Europea.

Cruz, M. \& Martínez, M. (2020). Origen y desarrollo de un índice de competencia experta: el coeficiente k". Revista Latinoamericana de Metodología de la Investigación Social ReLMIS, 19, año 10. 40-56.

Deng, R., Benckendorff, P. \& Gannaway, D. (2020). Learner engagement in MOOCs: Scale development and validation. British Journal of Educational Technology, 51(1), 245-262. doi:10.1111/bjet.12810.

Durán, M. (2019). Competencia Digital del Profesorado Universitario: Diseño y Validación de un Instrumento para la Certificación. Murcia. Universidad de Murcia.-Escuela Internacional de Doctorado. Tesis doctoral inédita.

Durán, M. C., Prendes, M.P.E. \& Gutiérrez, I. P. (2019). Certificación de la Competencia Digital Docente: propuesta para el profesorado universitario. RIED. Revista Iberoamericana de Educación a Distancia, 22(1), pp. 187-.205. Doi: http://dx.doi.org/10.5944/ried.22.1.22069.

Escudero-Nahón, A. \& Núñez-Urbina, A.A. (2020). Análisis crítico al término "masivo" en los MOOC: una Cartografía Conceptual. EDMETIC, Revista de Educación Mediática y TIC, 9(1), 188-212. doi: https://doi.org/10.21071/edmetic.v9i1.12252.

Fernández, E., Ordóñez, E., Morales, B. \& López, J. (2019). La competencia digital en la docencia universitaria. Barcelona: Octaedro.

Flores, C. \& Roig, R. (2016). Diseño y validación de una escala de autoevaluación de competencias digitales para estudiantes de pedagogía. Píxel..Bit, Revista de Medios y Educación, 12(48), 209-224. Doi: https://doi.org/10.12795/pixelbit.2016.i48.14

Evaluación de t-MOOC sobre competencias digitales docentes universitario mediante el juicio de expertos. Selección mediante el coeficiente de competencia experta. Cabero-Almenara, BarrosoOsuna, Palacios-Rodríguez, y Llorente-Cejudo.

Página 27 de 30 
RED. Revista de Educación a Distancia. Núm. 67, Vol. 21. Artíc. 2, 30-Sep-2021

DOI: http://dx.doi.org/10.6018/red.476891

Galicia, L., Balderrama, J. \& Edel, R. (2017). Validez de contenido por juicio de expertos: propuesta de una herramienta virtual. Apertura, 9(2), 42-53. Doi: http://dx.doi.org/10.18381/Ap.v9n2.993.

García-Peñalvo, F., Fidalgo-Blanco, A. \& Sein-Echaluce, M. (2018). An adaptive hybrid MOOC model: Disrupting the MOOC concept in higher education. Telematics and Informatics, 35(4), 1018-1030.

Gordillo, A., López-Pernas, S. \& Barra, E. (2019). Effectiveness of MOOCs for teachers in safe ICT use training. Comunicar, 61, DOI: 10.3916/C61-2019-09.

Gudmundsdottir, G. B. \& Hatlevic, O. E. (2018). Newly qualified teachers' professional digital competence: implications for teacher education. European Journal of Teacher Education, 41(2), 214--231.

Infante-Moro, A., Infante-Moro, J. \& Gallardo-Pérez, J. (2021). The acquisition of ICT skills at the university level: the case of the Faculty of Business Studies and Tourism of the University of Huelva. Píxel-Bit. Revista De Medios Y Educación, 60, 29-58. https://doi.org/10.12795/pixelbit.79471.

Landeta, J. (2002). El método Delphi: una técnica de previsión del futuro. Barcelona: Ariel.

Lázaro, J. L., Usart \& Gisbert, M. (2019). Assessing Teacher Digital Competence: the Construction of an Instrument for Measuring the Knowledge of Pre..Service Teachers. Journal of New Approaches in Educational Research, 8(1), 73 - 78. Doi: 10.7821/naer.2019.1.370.linguistica/files/articulosPDF/articulo_55002aca89c37.pdf.

Ljbojevic, M., Vaskovic, V., Stankovic, S. \& Vaskovic, J. (2015). El uso del vídeo complementario en la enseñanza multimedia como herramienta didáctica para incrementar la eficiencia del aprendizaje y la calidad de experiencia. Revista Mexicana de Bachillerato a Distancia, 13, 134-153.

López Gómez, E. (2018). El método Delphi en la investigación actual en educación: una revisión teórica y metodológica. Educación $X X 1,21(1), 17 \cdots 40 . \quad$ Doi: 10.5944/educXX1.15536.

Padilla-Hernández, A., Gámiz-'Sánchez, V. \& Romero-'López, A. (2019). Niveles de desarrollo de la competencia digital docente: una mirada a marcos recientes del ámbito internacional. Innoeduca. International Journal of Technology And Educational Innovation, 5(2), 140-150. Doi: 10.24310/innoeduca. 2019.v5i2.5600.

Malla, F. \& Zabala, I. (1978). La previsión del futuro en la empresa (III): el método Delphi.

Marín, V. (2017). Diseño y evaluación de un instrumento de competencia digital docente. Facultad de Educación, Universidad de las Islas Baleares, tesis doctoral no publicada.

Martínez, E. \& otros (2018). Identificación de las competencias específicas de los profesionales de enfermería en la atención al neonato en estado grave. Medisan, 22(2), 184-194.

Evaluación de t-MOOC sobre competencias digitales docentes universitario mediante el juicio de expertos. Selección mediante el coeficiente de competencia experta. Cabero-Almenara, BarrosoOsuna, Palacios-Rodríguez, y Llorente-Cejudo.

Página 28 de 30 
RED. Revista de Educación a Distancia. Núm. 67, Vol. 21. Artíc. 2, 30-Sep-2021

DOI: http://dx.doi.org/10.6018/red.476891

Martínez, E., Nadina, C., Sagaró, N, Urbina, O. \& Martínez, I. (2018). Identificación de las competencias específicas de los profesionales de enfermería en la atención al neonato en estado grave. Medisan, 22(2):184.

Mercado-del-Collado, R., Jácome-Avila, N., Ortega-Guerrer, J., Casillas-Alvarado, M. \& Ramírez-Martinell, A. (2019). Patrones de participación y logro en un MOOC de Saberes Digitales para Docentes en servicio de México. Research in Education and Learning Innovation Archives, 23,80--96. doi: 10.7203/realia/23/15904.

Nieto, E., Pech, S. \& Callejas, A. (2017). Evaluación de la competencia digital docente. TIC y enseñanza de idiomas. En Sumozas, R. \& Nieto, E. (coords.). Evaluación de la competencia digital docente, 17--33.

Osuna-Acedo, S., Marta-Lazo, C. \& Frau-Meig, D. (2018). De sMOOC a tMOOC, el aprendizaje hacia la transferencia profesional: El proyecto europeo ECO. Comunicar, 55, 105-114. DOI https://doi.org/10.3916/C55-2018-10.

Padilla, A., Gámiz, V. \& Romero, M.A. (2019). Validación del contenido de un guion de entrevista sobre la competencia digital docente en Educación Superior. Revista lbérica de Sistemas e Tecnologias de Informação, 32(6), 1-16.

Palacios, F., Huertas, C. \& Gómez, M.E. (2020). MOOCs: Origins, Concept and Didactic Applications: A Systematic Review of the Literature (2012-2019). Technology, Knowledge and Learning, https://doi.org/10.1007/s10758-019-09433-6.

Peraza, M., Armenta, A. \& Hernández, S. (2019). Juicio de expertos para la validación de un proyecto formativo. Revista Electrónica Desafíos Educativos, 5, 24-34.

Pilli, O. \& Admiraal, W. (2016). A Taxonomy of Massive Open Online Courses. Contemporary Educational Technology, 7(3), 223-240.

Recio, F., Silva, J. \& Abricot, N. (2020). Análisis de la Competencia Digital en la Formación Inicial de estudiantes universitarios: Un estudio de meta-análisis en la Web of Science. Píxel-Bit. Revista De Medios Y Educación, 59, 125-146. https://doi.org/10.12795/pixelbit.77759

Robles, P. \& Rojas, M.D.C. (2015). La validación por juicio de expertos: dos investigaciones cualitativas en Lingüística aplicada. Revista Nebrija de Lingüística Aplicada, 18. Recuperado de https://www.nebrija.com/revistaw.

Rodríguez, M. D. M., Méndez, V. G., \& Martín, A. M. R. M. R. (2018). Alfabetización Informacional Y Competencia Digital En Estudiantes De Magisterio. Profesorado, Revista de Currículum y Formación Del Profesorado, 22(3), 253-270. Doi: https://doi.org/10.30827/PROFESORADO.V22I3.8001

Rodríguez-García, A., Trujillo, J.M. \& Sánchez, J. (2029). Impacto de la productividad científica sobre competencia digital de los futuros docentes: aproximación bibliométrica en Scopus y Web of Science. Revista Complutense de Educación, 30(2), 623-646.

Ruiz, A., Medina, M., Pérez, E., \& Medina, A. (2020). University teachers' training: the Digital Competence. Píxel-Bit. Revista De Medios $Y$ Educación, 58, 181-215. https://doi.org/10.12795/pixelbit.74676.

Ruiz-Palmero, J., López-Álvarez, D. \& Sánchez-Rivas, E. (2021). Revisión de la producción

Evaluación de t-MOOC sobre competencias digitales docentes universitario mediante el juicio de expertos. Selección mediante el coeficiente de competencia experta. Cabero-Almenara, BarrosoOsuna, Palacios-Rodríguez, y Llorente-Cejudo.

Página 29 de 30 
RED. Revista de Educación a Distancia. Núm. 67, Vol. 21. Artíc. 2, 30-Sep-2021

DOI: http://dx.doi.org/10.6018/red.476891

científica sobre MOOC entre 2016 y 2019 a través de SCOPUS. Píxel-Bit. Revista De Medios Y Educación, 60, 95-107. https://doi.org/10.12795/pixelbit.77716

Sahasrabudhe, V. \& Kanungo, S. (2014). ppropriate media choice for e-learning effectiveness: Role of learning domain and learning style. Computers \& Education 76, 237-249. http://dx.doi.org/10.1016/j.compedu.2014.04.006.

Salazar, E. \& Gibrán, L. (2019). Construct Validity and Reliability of a Rubric for the Evaluation of Digital Competences from the Socioformative Approach. FORHUM. International Journal of Social Sciences and Humanities, 1(1), 82-99, Doi: dx.doi.org/10.35766/jf1911.

Salim, P. \& Luo, T. (2019). Factors contributing to student retention in online learning and recommended strategies for improvement: a systematic literature review. Journal of Information Technology Education Research, 18, 19-57. https://doi.org/10.28945/4182.

Silva, J. (2017). Un modelo pedagógico virtual centrado en las E-actividades. RED. Revista de Educación a Distancia, 53, 10, 1-20, http://www.um.es/ead/red/silva.pdf.

Silva, J., Morales, M. J., Lázaro, J. L. \& Gisbert, M. (2019). La competencia digital docente en formación inicial: Estudio a partir de los casos de Chile y Uruguay. Archivos Analíticos de Políticas Educativas, 27(93). Doi: https://doi.org/10.14507/epaa.27.3822.

Tourón, J., Martín, D., Navarro, E., Pradas, S. \& Íñigo, V. (2018). Validación de constructo de un instrumento para medir la competencia digital docente de los profesores (CDD). Revista Española de Pedagogía, 76(269), 25-54. Doi:

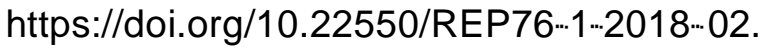

Witkin, B. R. \& Altschuld, J. W. (1995). Planning and conducting needs assessment: A practicalguide. Thousand Oaks, Sage.

Zawacki-Richer, O., Bozkurt, A., Alturki, U. \& Aldraiweesh, A. (2018). What Research Says About MOOCs - An Explorative Content Analysis. International Review of Research in Open and Distributed Learning, 19(1), 242-259.

Zhao, S. \& Song, J. (2020). Students' Perceptions of a Learning Support Initiative for bMOOCs. iJET, 15(21), 179-194.

Zhou, M. (2016). Chinese university students' acceptance of MOOCs: A self-determination perspective. Computers \& Education 92-93, 194-203.

Evaluación de t-MOOC sobre competencias digitales docentes universitario mediante el juicio de expertos. Selección mediante el coeficiente de competencia experta. Cabero-Almenara, BarrosoOsuna, Palacios-Rodríguez, y Llorente-Cejudo.

Página 30 de 30 